ORIENTAL JOURNAL OF
ISSN: 0974-6471
June 2017,
COMPUTER SCIENCE \& TECHNOLOGY

\title{
Agile Practices in Indian Organizations
}

\author{
UMA KUMARI \\ Jagannath University, Jaipur, India \\ Corresponding author Email: umasecd@gmail.com \\ http://dx.doi.org/10.13005/ojcst/10.02.24
}

(Received: April 29, 2017; Accepted: May 29, 2017)

\begin{abstract}
Agile software development is a conceptual framework that promotes development using iterations in whole life cycle of the project development. In Indian organizations agile is in its embryo stage. In software development agile means quick moving. For the customer's satisfaction and to cope up with customer's frequent changed requirements, heavyweight methodologies are kicked. To produce high quality software products and to meet stakeholder's requirements are the two major challenges in software development. Today, little is known about which agile methods are being used by Indian companies and whether people are satisfied or not with the methods that they are using. This is due to the lack of objective surveys on the subject. To accomplish this, an independent online web based survey, interview survey and questionnaire survey was conducted. Motive was to find the total percentage of users in India, who are using agile and it was tried to find that does it increase the productivity, quality and cost of software. Results are proved using statistical one way ANOVA method. Different Hypothesis that are designed are

Hypothesis I: Production gets increased on using different methodologies of agile instead of heavyweight methods.

Hypothesis II:Quality gets increased on using different methodologies of agile instead of heavyweight methods.

Hypothesis III: Cost gets reduced on using different methodologies of agile instead of heavyweight methods. Categories and Subject Descriptors: Agile, heavyweight methods, XP, Scrum, DSDM, FDD, ASD
\end{abstract}

Keywords: Agile; heavyweight methods; XP; Scrum; DSDM; FDD; ASD; ANOVA; iterative; traditional methods.

\section{INTRODUCTION}

In India little is known about which agile methodologies companies are using and which type of need (out of agile or heavyweight) software companies have nowadays. Due to the lack of any survey, vendors as well as companies are facing problems regarding adaptation of an appropriate agile methodology. Goal of surveys was not only to collect statistics about usage and needs desired by companies but also to gain understanding of features. Understanding of features was done by means of gathering comments from many company representatives. The software market is becoming more dynamic which can be easily seen from frequent changes in the needs of customers. Hence, there is a need to quickly respond to these changes. For software companies this means that they have 
to become agile with the objective of developing features like very short lead-time and high quality.

Dynamically changing environments make changes to requirements in the software development process an inevitable task. Poor requirements and changes to requirements are major elements that cause software project failures ${ }^{1}$. To cope up with this agile is trying to meet the demands of customers. Agile is defined as

An iterative and incremental (evolutionary) approach to software development which is performed in a highly collaborative manner by selforganizing teams within an effective governance framework with "just enough" ceremony that produces high quality solutions in a cost effective and timely manner which meets the changing needs of its stakeholders ${ }^{2}$.

\section{Related Work in Agile}

Due to constant changes in the technology and business environments, it is a challenge for traditional software development methods to create a complete set of requirements up front [3]. Recently, more attention is paid to agility because of the constant change in requirements and to produce cost effective software.

Agile methodologies began its journey in the mid-1990s, when software methodologies and techniques such as Extreme Programming ${ }^{4}$,

Taking Hypothesis "Production increases on using different methodology of Agile instead of heavyweight methods". This can be proved by taking one-way ANOVA. The frequency table can be constructed as below:

Table Annex 1.1: Frequency and Agile methodology

\begin{tabular}{lccccc}
\hline & A1 & A2 & A3 & A4 & A5 \\
\hline XP & 0 & 2 & 0 & 0 & 3 \\
Scrum & 1 & 1 & 2 & 9 & 12 \\
DSDM & 0 & 0 & 1 & 0 & 1 \\
FDD & 0 & 1 & 1 & 0 & 2 \\
Lean & 0 & 0 & 0 & 2 & 5 \\
Others & 3 & 2 & 7 & 8 & 0 \\
\hline
\end{tabular}

Scrum $^{5}$, Crystal Family of Methodologies ${ }^{6}$, Dynamic Systems Development Method (DSDM) ${ }^{7}$, Adaptive Software Development (ASD) ${ }^{8}$, and Feature-Driven Development (FDD) ${ }^{9}$ began to emerge.

T. Dyba, \& T. Dingsoyr summarizes the differences between agile development and traditional development on the basis of an unpredictable world, as well as emphasizing the value competent people and their relationships to software development ${ }^{10}$.

\section{To calculate the F-ratio}

Step 1: Mean within each group $\_1=1 / 6 \Sigma Y 1 \mathrm{i}=$ 0.67

$\_2=1 / 6 \Sigma Y 2 i=1$

$\_3=1 / 6 \Sigma Y 3 i=1.833 \_4=1 / 6 \Sigma Y 4 i=3.167 \_5=$ $1 / 6 \Sigma Y 5 i=3.833$

Step 2: Overall mean

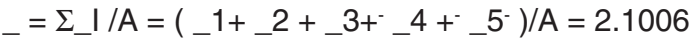

Where $A$ is number of groups.

Step 3: "Between group" sum of squares

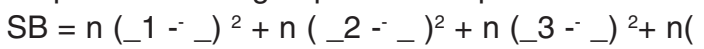
4-- $)^{2}+\mathrm{n}\left(\mathrm{Y} 5\right.$ - $\left._{-}\right) 2$

$\mathrm{SB}=38.36$

Where $n$ is number of data values per group.

The between-group degrees of freedom are one less than the number of groups. $f b=5-1$ $=4$

MSB $=38.36 / 4=9.59$ (So the between group mean square value)

A model for integrating Scrum and XP was proposed by Zaigham Mushtaq in 2012 for good project management paradigm and to produce quality software product that is aligned with customer requirements and company objectives ${ }^{11}$. But this model is not tested using industrial environments.

According to a model suggested by Boehm and Turner, Carlton, Hakan et al. consider their model a useful starting point for generating hypothesis for future research, which could lead to generalize and actionable recommendation for 
scientific software development community in future. According to them agile is well suited for exploratory, iterative and collaborative but agile methodologies might not be applicable in all situations pertaining to scientific software development projects ${ }^{12}$.

\section{Brief Overview of Heavyweight Methods and Agile}

Heavyweight methods are also known as traditional methods. These methodologies are based on a series of steps, such as requirements analysis, coding, testing, deployment and maintenance of project. Heavyweight methodologies are focused on planning everything from the start of a project.

Step 4: "Within - group" sum of squares.

Table Annex 1.2: Within Group Analysis

\begin{tabular}{|c|c|c|c|c|}
\hline A1 & $\mathrm{A} 2$ & A3 & A4 & A5 \\
\hline $0-0.67=-$ & $2-1$ & $0-1.833=-$ & $0-3.167=-$ & $3-3.833=$ \\
\hline 0.67 & $=1$ & 1.833 & 3.167 & 0.833 \\
\hline $1-0.67=$ & $1-1$ & 2- & $9-$ & $12-$ \\
\hline 0.33 & $=0$ & $\begin{array}{c}833=0 \\
.167\end{array}$ & $\begin{array}{c}3.167=5.8 \\
33\end{array}$ & $\begin{array}{c}3.833= \\
8.167\end{array}$ \\
\hline $0-0.67=-$ & $0-1$ & $1-1.833=-$ & $0-3.167=-$ & $1-3.833=-$ \\
\hline 0.67 & $=-1$ & 0.833 & 3.167 & 2.833 \\
\hline $0-0.67=-$ & $1-1$ & $1-1.833=-$ & $0-3.167=-$ & $2-3.833=-$ \\
\hline 0.67 & $=0$ & 0.833 & 3.167 & 1.833 \\
\hline $0-0.67=-$ & $0-1$ & $0-1.833=-$ & $2-$ & $5-$ \\
\hline 0.67 & $=-1$ & 1.833 & $\begin{array}{c}3.167=1.1 \\
67\end{array}$ & $\begin{array}{c}3.833= \\
2.833\end{array}$ \\
\hline $3-0.67=$ & $2-1$ & 7- & $8-$ & $0-3.833=-$ \\
\hline 2.33 & $=1$ & $\begin{array}{c}1.833= \\
5.167\end{array}$ & $\begin{array}{c}3.167= \\
4.8 \\
33\end{array}$ & 3.833 \\
\hline
\end{tabular}

Within-group sum of squares is the sum of squares of all 30 values in this table.

$\mathrm{S} w=(-0.67) 2+(0.33) 2+(-0.67) 2+(-0.67) 2+$ $(-0.67) 2+(2.33) 2+(1) 2+(0) 2+(-1) 2+(0) 2+(-1) 2$ $+(1) 2+(-1.833) 2+(0.167) 2+(-0.833) 2+(-0.833) 2$ $+(-1.833) 2+(5.167) 2+(-3.167) 2+(5.833) 2$ $+(-3.167) 2+(-3.167) 2+(1.167) 2+(4.833) 2+$ $(-0.833) 2+(8.167) 2+(-2.833) 2+(-1.833) 2+$ $(2.833) 2+(-3.833) 2=236.4983$

$\mathrm{MSw}=\mathrm{S}$ w/ f w $=236.4983 / 25=9.46$

Step 5: F-ratio is

$\mathrm{F}=\mathrm{MSB} / \mathrm{MSw}=1.013$

Fcrit $(4,25)=2.758$ at $\alpha=0.05$
These methodologies are very documentation centric. Producing high quality software products and meeting the stakeholder's requirements are major challenges in software engineering. Dynamically changing environments make changes to requirements in the software development process an inevitable task. Poor requirements and changes to requirements are major elements that cause software project failures ${ }^{1}$. Due to all these

2. Taking hypothesis "Quality get increases on using different methodology of Agile instead of heavyweight methods".

Table Annex 2.1

\begin{tabular}{lccccc}
\hline & A1 & A2 & A3 & A4 & A5 \\
& & & & & \\
XP & 0 & 2 & 0 & 1 & 2 \\
Scrum & 2 & 1 & 1 & 8 & 13 \\
DSDM & 0 & 0 & 1 & 0 & 1 \\
FDD & 0 & 0 & 1 & 3 & 0 \\
Lean & 0 & 0 & 0 & 2 & 4 \\
Others & 5 & 4 & 4 & 7 & 0 \\
\hline
\end{tabular}

To calculate F-ratio

Step 1: Mean within each group $\_1=1 / 6 \Sigma Y 1 \mathrm{i}=$ 1.667

$\_2=1 / 6 \Sigma Y 2 i=1.667 \_3=1 / 6 \Sigma Y 3 i=1.667 \_4=$ $1 / 6 \Sigma Y 4 i=3.5 \_5=1 / 6 \Sigma Y 5 i=3.333$

Step 2: Overall mean

_ $=\Sigma \_$I /A = ( _ 1+_ $\left.2+\_3+\_4+\_5\right) / A=2.3668$ Where $A$ is number of groups.

Step 3: "Between group" sum of squares

$\mathrm{SB}=\mathrm{n}\left(\_1\right.$ - _ $) 2+\mathrm{n}\left(\right.$ _ $_{-}$- $) 2+\mathrm{n}\left(\_3\right.$ - _ $) 2+\mathrm{n}($ 4- _ $) 2+n\left(Y 5-_{-}\right) 2$

$\mathrm{SB}=9.65635$

Where $\mathrm{n}$ is number of data values per group.

The between-group degrees of freedom are one less than the number of groups. $f b=5-1=4$

so the between group mean square value is MSB $=9.65635 / 4=2.414$ 
problems, to cope with current market needs and to save cost and time agile methods are being adopted by companies.

Agile started its journey when 17 representatives of different agile method experts, met at Utah and formulated agile manifesto ${ }^{13}$ which consists of 12 principles and 4 values. In agile software development, process does not follow defined steps, but it uses very short iterations of about 2-4 weeks which focus on producing working software rather than heavy documentation. Agile also allows requirements to emerge throughout the development process and it allows changes

Step 4: "Within- group" sum of squares

Table Annex 2.2

\begin{tabular}{lcccc}
\hline & & & & \\
A1 & A2 & A3 & A4 & A5 \\
\hline $0-1.667=$ & $2-1.667=$ & $0-1.667=$ & $1-3.5=$ & $-2-3.333=-$ \\
-1.667 & 0.333 & -1.667 & 2.5 & 1.333 \\
$2-1.667=$ & $1-1.667=$ & $1-1.667=$ & $8-3.5=$ & $13-3.333=$ \\
0.333 & -0.667 & 0.667 & 4.5 & 9.667 \\
$0-1.667=$ & $0-1.667=$ & $1-1.667=$ & $0-3.5=$ & $-1-3.333=-$ \\
-1.667 & -1.667 & 0.667 & 3.5 & 2.333 \\
$0-1.667=$ & $0-1.667=$ & $1-1.667=$ & $3-3.5=$ & $0-3.333=-$ \\
-1.667 & -1.667 & 0.667 & 0.5 & 3.333 \\
$0-1.667=$ & $0-1.667=$ & $0-1.667=$ & $2-3.5=$ & $-4-3.333=$ \\
-1.667 & -1.667 & -1.667 & 1.5 & 0.667 \\
$5-1.667=$ & $4-1.667=$ & $4-1.667=$ & $7-3.5=$ & $0-3.333=-$ \\
3.333 & 3.333 & 3.333 & 2.5 & 3.333 \\
\hline
\end{tabular}

Within-group sum of squares is the sum of squares of all 30 values in this table.

$\mathrm{S} w=(-1.667) 2+(0.333) 2+(-1.667) 2+(-1.667) 2$ $+(-1.667) 2+(3.333) 2+(0.333) 2+(-0.667) 2+$ $(-1.667) 2+(-1.667) 2+(-1.667) 2+(3.333) 2+$ $(-1.667) 2+(0.667) 2+(-0.667) 2+(0.667) 2+$ $(-1.667) 2+(3.333) 2+(-2.5) 2+(4.5) 2+(-3.5) 2$

$+(0.5) 2+(1.5) 2+(2.5) 2+(1.333) 2+(9.667) 2$

$+(2.333) 2+(3.333) 2+(0.667) 2+(3.333) 2$ $=248.3893$

$M S w=S w / f w=248.3893 / 25=9.935$

Step 5: F-ratio is

$\mathrm{F}=\mathrm{MSB} / \mathrm{MSw}=2.414 / 9.935=0.2429$ Fcrit $(4,25)$

$=2.758$ at $\alpha=0.05$ to requirements even late into the project with minimum impact on software functionality and quality of the delivered product. It has the properties like iterative development having short iterations, working versions at completion of each iteration,

3. Taking Hypothesis "Cost reduces on using different methodology of Agile instead of heavyweight methods".

It can be proved as follows:

Table Annex 3.1

\begin{tabular}{lccccc}
\hline & A1 & A2 & A3 & A4 & A5 \\
XP & 2 & 2 & 0 & 1 & 0 \\
Scrum & 14 & 2 & 0 & 1 & 3 \\
DSDM & 0 & 7 & 1 & 0 & 0 \\
FDD & 1 & 1 & 1 & 1 & 0 \\
Lean & 5 & 1 & 0 & 0 & 1 \\
Others & 2 & 10 & 7 & 0 & 0 \\
\hline
\end{tabular}

To calculate F-ratio

Step 1: Mean within each group

$\_1=1 / 6 \Sigma Y 1 i=(2+14+0+1+5+2) / 6=24 / 6=4$

$2=1 / 6 \Sigma Y 2 i \quad=(2+2+7+1+1+10) / 6=23 / 6=$

3.833

$3=1 / 6 \Sigma \mathrm{Y} 3 \mathrm{i} \quad=(0+0+1+1+0+7) / 6=9 / 6=1.5$

$-4=1 / 6 \Sigma Y 4 i \quad=(1+1+0+1+0+0) / 6=3 / 6=0.5$

$-5=1 / 6 \Sigma Y 5 i \quad=(0+3+0+0+1+0) / 6=4 / 6=$ 0.667

Step 2: Overall mean

_ $=\Sigma \_\mathrm{I} / \mathrm{A}=\left(\right.$ _ $\left.1+\_2+\_3+\_4+\_5\right) / \mathrm{A}=(4+3.833+$ $1.5+0.5+0.667) / 5=10.5 / 5=2.1$ Where $A$ is number of groups.

Step 3: "Between group" sum of squares

$\mathrm{SB}=\mathrm{n}\left(\_1\right.$ - _ $) 2+\mathrm{n}\left(\right.$ _ $_{-}$- $) 2+\mathrm{n}\left({ }_{3}\right.$ - _ $) 2+\mathrm{n}($ -4- -$) 2+n\left(Y 5-\_\right) 2$

$\mathrm{SB}=6(4-2.1) 2+6(3.833-2.1) 2+6(1.5-$ $2.1) 2+6(0.5-2.1) 2+6(0.667-2.1) 2=21.66$ $+17.98+2.16+15.36+12.29=69.45$ Where $n$ is number of data values per group.

The between-group degrees of freedom are one less than the number of groups. $f b=5-1=4$ so the between group mean square value is MSB $=69.45 / 4=17.36$ 
fully integrated and tested methodology, adaptable and people-centric.

\section{RESULTS}

Based on the web based survey, questionnaire and interview method, following

Step 4: "Within- group" sum of squares.

\begin{tabular}{lcccc}
\hline \multicolumn{5}{c}{ Table Annex 3.2 } \\
\hline A1 & A2 & A3 & A4 & A5 \\
\hline $2-4=-2$ & $2-3.833$ & $0-1.5$ & $1-0.5=$ & $0-0.667$ \\
& $=1.833$ & $=-1.5$ & 0.5 & $=-0.667$ \\
$14-4=10$ & $2-3.833$ & $0-1.5$ & $1-0.5=$ & $3-0.667$ \\
& $=1.833$ & $=-1.5$ & 0.5 & $=2.33$ \\
$0-4=-4$ & $7-3.833$ & $1-1.5$ & $0-0.5=$ & $0-0.667=$ \\
& $=4.17$ & $=0.5$ & -0.5 & -0.667 \\
$1-4=-3$ & $1-3.833$ & $1-1.5$ & $1-0.5=$ & $0-$ \\
& $=-2.833$ & $=0.5$ & 0.5 & $0.667=-$ \\
& & & & 0.667 \\
$5-4=1$ & $1-3.833$ & $0-1.5$ & $0-0.5=$ & $1-$ \\
& $=-2.833$ & $=-1.5$ & -0.5 & $0.667=-$ \\
& & & & 0.667 \\
$2-4=-2$ & $10-3.833$ & $7-1.5$ & $0-0.5=$ & $0-0.667=$ \\
& $=6.17$ & $=5.5$ & -0.5 & -0.667 \\
\hline
\end{tabular}

The within-group sum of squares is the sum of squares of all 30 values in this table.

$\mathrm{S} w=(-1.667) 2+(0.333) 2+(-1.667) 2+(-1.667) 2$ $+(-1.667) 2+(3.333) 2+(0.333) 2+(-0.667) 2+$ $(-1.667) 2+(-1.667) 2+(-1.667) 2+(3.333) 2+$ $(-1.667) 2+(0.667) 2+(-0.667) 2+(0.667) 2+$ $(-1.667) 2+(3.333) 2+(-2.5) 2+(4.5) 2+(-3.5) 2$ $+(0.5) 2+(1.5) 2+(2.5) 2+(1.333) 2+(9.667) 2+$ $(2.333) 2+(3.333) 2+(0.667) 2+(3.333) 2=2.7789$ $+0.1108+2.7789+2.7789+2.7789+11.1089$ $+0.1108+0.4449+2.7789+2.7789+2.7789+$ $11.1089+2.7789+0.4449+0.4449+$

$0.4449+2.7789+11.108+6.25+20.25+12.25$ $+0.25+2.25+6.25+1.7769+93.45+5.442+$ $11.108+0.4449+11.108=11.2264+17.2224+17$ $.5565+40.8318+22.7769+121.5529=248.3893$

$\mathrm{MSw}=\mathrm{S} w / \mathrm{f} w=248.3893 / 25=9.935$

Step 5

The F-ratio is

$\mathrm{F}=\mathrm{MSB} / \mathrm{MSw}=17.36 / 9.935=1.747$ Fcrit $(4,25)$ $=2.758$ at $\alpha=0.05$ results were obtained. The survey covers 70 responses by these modes. The online survey was available at http://www.surveyact.com/s/ Szjtjuw1AZ5DJuii in month of June to August 2013.

\section{Annexure II}

Table 1: Effectiveness of Agile in Case of Productivity

How Effective Agile $\quad$ Frequency
Methods are in
Case of Productivity

Much Lower 4

Somewhat Lower 7

No Change 10

Somewhat Higher $\quad 20$

Much Higher 25

Table 2: Effectiveness of Agile in Case of Quality

How Effective Agile Frequency
Methods are in
Case of Quality

Much Lower 7

Somewhat Lower 8

No Change 7

Somewhat Higher $\quad 21$

Much Higher 22

Table 3: Effectiveness of Agile in Case of Cost of System Development

How Effective Agile Frequency
Methods are in
Case of Cost
of System
Development

$\begin{array}{lc}\text { Much Lower } & 26 \\ \text { Somewhat Lower } & 23 \\ \text { No Change } & 9 \\ \text { Somewhat Higher } & 3 \\ \text { Much Higher } & 4\end{array}$




\section{Hypothesis Designing and proof}

Following Hypothesis are designed and proveed using F-test and one way ANOVA method. Values obtained by researcher are displayed using frequency tables (Annexure II). This analysis is done on the data obtained by researcher by different modes such as questionnaire, interview and wed based survey.Table 1, Table 2 and Table 3 are constructed accordingly

\section{Hypothesis I}

Production increases on using different methodology of Agile instead of heavyweight methods.

\section{Proof}

Researcher obtained $0.2429<2.758$, where 0.2429 is calculated value and 2.758 is table value. The results are insignificant at the $5 \%$ significance level. There is no evidence that expected values in five groups differ.

The calculated value of $F$ is less than the table value then it is insignificant and hypothesis is accepted. In above case the calculated value of the $F(1.013)$ is less than the table value (2.758) hence it is insignificant at $5 \%$ level of significance and Hypothesis is accepted (Annexure I).

\section{Hypothesis II}

Quality gets increased on using different methodologies of agile instead of heavyweight methods.

\section{Proof}

Researcher obtained $0.2429<2.758$, where 0.2429 is calculated value and 2.758 is table value. The results are insignificant at the $5 \%$ significance level. There is no evidence that expected values in five groups differ.

So, the calculated value of $F$ is less than the table value then it is insignificant and hypothesis is accepted. In above case the calculated value of the $F(0.2429)$ is less than the table value (2.758) hence it is insignificant at $5 \%$ level of significance and Hypothesis is accepted (Annexure I).

\section{Hypothesis III}

Cost gets reduced on using different methodologies of agile instead of heavyweight methods.

\section{Proof}

Researcher obtained $1.747<2.758$ where 1.747 is calculated value and 2.758 is table value. The results are insignificant at the $5 \%$ significance level. There is no evidence that expected values in five groups differ.

So, the calculated value of $F$ is less than the table value then it is insignificant and hypothesis is accepted. In above case the calculated value of the $F(1.747)$ is less than the table value (2.758) hence it is insignificant at $5 \%$ level of significance and hypothesis is accepted (Annexure I).

So, according to findings it has been observed that quality and production gets increased on using agile and cost gets decreases on using agile (Annexure I).

\section{CONCLUSION}

In this paper, the results of survey on agile methodology in Indian organizations are presented. In the traditional software development methodologies, the lack of user input, incomplete requirements, and changing requirements are some of the major reasons for not to deliver all planned functionality on schedule and within budget.

An interesting fact that is noticed is that a company mostly developing small projects is enjoying a good amount success in using agile methods. It is also observed that the companies are not really being encouraged in terms of using a variety of agile methods [14]. This is because of lack of sound proficiency in this field. It is therefore important that empirical research and investigation should be carried in this field. It can be understood that agile methods are highly practical oriented. Application of different practices of agile methodologies differs from company to company. 


\section{REFERENCES}

1. E. O. Oyeyipo, An empirical study of requirements management in an agile-scrum development

environment, Dissertation, 2011, pp. x, xi

2. http://www.agilemodeling.com/essays/ agileSoftwareDevelopment.htm

3. Parikshit Joshi,Ashish Aggarwal and Shivani Goel. 2013.Communication issues in agile methodology: A Surver. IJLRST,VOL 2(ISSUE 4), PAGE 15-20

4. K. Beck, \& C. Andres, Extreme Programming Explained: Embrace Change. Second Edition. Addison-Wesley. Boston, 2004, pp. 189.

5. K. Schwaber, Agile Project Management with Scrum. Microsoft Press, Washington, 2004, pp. 163.

6. A. Cockburn, Surviving Object-Oriented Projects, Addison-Wesley, Reading Mass, 1998, pp. 250.

7. J. Stapleton, DSDM: Business Focused Development. Second Edition. Addison Wesley, London, 2003, pp. 239.

8. J. A. Highsmith, Adaptive Software Development: A Collaborative Approach to Managing Complex Systems, New York: Dorset House, 2000, ISBN 0-932633- 40-4, pp. 392.

9. P. Coad, E. LeFebvre \& J. De Luca, Java
Modeling In Color With UML: Enterprise Components and Process, Prentice Hall,1999, pp. 221.

10. T. Dyba, \& T. Dingsoyr, What do we know about agile software development? Software, IEEE, 26(5), 2009, pp. 6-9.

11. Z. Mushtaq, \& M.R.J. Qureshi, Novel hybrid model:Integrating Scrum and XP, I.J. Information Technology and Computer Science(IJITCS), June 2012, issue VI, pp 39-44

12. C. A. Crabtree, A. G. Koru, C. Seamon, \& H. Erdogmus, An Empirical Characterization of Scientific Software Development Projects According to the Boehm and Turner Model: a Progress Report, SECSE'09, ICSE' 09 workshop, 23 May 2009, Vancouver, Canada.

13. http://www.agilemanifesto.org/

14. U. Kumari, Agile survey: Indian organizations, International Journal of Engineering Research and Technology, Vol2(issue 12) (IJERT), pp. 1450-1454 